\title{
Desarrollo agrícola y uso de agroquímicos en el valle de Mexicali
}

\author{
JoséA. Moreno Mena* \\ Mercedes Gema López Limón**
}

\begin{abstract}
Resumen. Este trabajo se presenta una propuesta de periodización histórica del uso de plaguicidas y otros agroquímicos en el valle de Mexicali, tomando en cuenta la evolución del desarrollo agrícola en la región y su contexto. El uso de una diversidad de productos químicos en la agricultura ha sido resultado de los modelos tecnológicos industriales que se han adoptado desde principios de siglo, especialmente de las sugerencias de la "revolución verde". Se resalta la importancia que están teniendo las hortalizas como consumidoras de plaguicidas. También se observa que a pesar de que por varias décadas se han usado agroquímicos en la agricultura del valle mexicalense, son escasas las investigaciones sobre su impacto en la salud pública y en el medio ambiente. Lo que se conoce por estudios realizados en zonas agrícolas que utilizan estos productos es preocupante, por lo que se propone la necesidad de realizar estudios que tiendan a profundizar en esa problemática.

Palabras clave: Salud pública, pesticidas, valle de Mexicali.
\end{abstract}

\begin{abstract}
A bstract. This article presents a proposal of historical periodization regarding the use of pesticides and other agrochemicals products in the Valley of Mexicali, taking into account the evolution of regional agricultural development and its context. The use of diverse chemical products in agriculture was the result of industrial an technological models which were adopted at the beginning of the twentieth century and, in particular, as a resul of suggestions arising out of the "green revolution". We highligt the importance of vegetables as a primary consumer of pesticides. We also note that, in spite of the use of agrochemical products in the Valley of Mexicali for various decades, little research has been done regarding the effects on public health and the environment. What little information has been gathered from studies carried out in agricultural zones utilizing these products is alarming. Therefore, we underline the need to carry out research that delves more deeply into long-term effects of the use of such products.
\end{abstract}

Keywords: Public health, pesticides, valley of Mexicali

* Investigador del Instituto de Investigaciones Sociales de la Universidad Autónoma de Baja California y becario de PROMEP para estudios de doctorado. Correo electrónico: jmoreno@.ens.uabc.mx.

** Investigadora del Instituto de Investigaciones Sociales de la Universidad Autónoma de Baja California. Correo electrónico: glopez@uabc.mx 


\section{Introducción}

El uso de los agroquímicos ${ }^{1}$ en el valle de Mexicali está íntimamente relacionado con los modelos agrícolas que se han venido adoptando desde su origen como zona productora. El tipo de productos químicos utilizados para contrarrestar las plagas ha observado una evolución similar a la tendencia general en la agricultura estadounidense, en particular en las zonas de California, aunque con diferencias importantes en cuanto a las reglamentaciones.

La relación de vecindad con el Valle Imperial californiano favoreció el fácil acceso a la tecnología moderna, especialmente a los agroquímicos. Del lado mexicano, la adopción de nuevos plaguicidas y fertilizantes que aparecieron en los valles estadounidenses se presentó de manera casi inmediata, sobre todo en los cultivos de exportación. Sin embargo, a diferencia denuestros vecinos, se registra durante varios años la aplicación de al gunos plaguicidas que han sido prohibidos o severamente restringidos en Estado Unidos y otros países, observándose en especial en cultivos para el mercado interno.

A pesar de la gran diversidad de compuestos químicos utilizados desdequeseinició la agricultura como actividad comercial en el Valle deM exicali, podemos observar el uso predominantedecierta clasede plaguicidas durante diferentes etapas.

Este trabajo tiene el propósito de presentar una propuesta de periodización histórica sobreel uso de los agroquímicos, tomando en cuenta el proceso evolutivo del desarrollo agrícola en el contexto socioeconómico que siguió el vallede Mexicali en el siglo xx.

Los cuatro periodos sugeridos tienen como eje fundamental el uso general izado de una determinada familia dequímicos en la agricultura, que por lo regular se definen basándose en su estructura y composición química. También se presenta una serie de caracterís-

\footnotetext{
${ }^{1}$ Por agroquímicos se entiende todas aquellas sustancias químicas que se utilizan en la producción agrícola para enriquecer los cultivos (fertilizantes) o eliminar a los insectos, hongos, arañas y hierbas consideradas como plagas (plaguicidas). Existen otros compuestos que cumplen diversas funciones en la agricultura, como los reguladores de crecimiento, feromonas, desecantes, etcétera.
} 
ticas del desarrollo social y agrícola del periodo como contexto. Al final, atendiendo a estudios realizados en zonas semejantes de agricultura comercial, se hace un recuento de los daños que ocasiona la utilización de los agroquímicos en la salud humana, tanto en sus efectos agudos (intoxicaciones) como en el de daños a mediano y largo plazo (efectos crónicos). En el trabajo se menciona la escasez de estudios sobre el impacto de los agroquímicos en la salud pública y el medio ambiente en el Valle de Mexicali y la necesidad de realizarlos.

\section{El uso de los agroquímicos en el valle de Mexicali, periodo 1912-1948}

\section{Características del periodo}

A principios del siglo xx, encontramos un valleagrícola prácticamente virgen, formando parte de una unidad geográfica compartida con el Vallelmperial del vecino estado deCal ifornia, Estados Unidos. Hay un origen común en el surgimiento de estos valles, separados artificialmente por la división política entreambos países. Por sus características topográficas, las aguas del Río Colorado en diferentes etapas históricas y geológicas permitieron la formación de terrenos aluvial es sumamentericos. Como factores sociales en la creación dela región seencuentra, por un lado, el proceso de acumulación originaria de capital que se desarrolló en México en el siglo xIx, con las leyes de Reforma y la desamortización de los bienes del clero y de las comunidades indígenas, queincorporaron así la tierra al mercado; y por el otro, la expansión del capitalismo en el suroestedeEstados Unidos, que en diferentes momentos del siglo xIx avanzó en la búsqueda de colonizar esta zona, factores que generaron las condiciones para el surgimiento de la región agrícola en el delta del Río Colorado (EstreIla, 1980).

A nteriormente, en el valle de Mexicali (en adelante VMxl) existía en las orillas de los ríos una agricultura incipiente y temporalera de autoconsumo y familiar, practicada por las tribus seminómadas que habitaban la región. También se habían hecho intentos por cultivar 
la tierra por parte de unos cuantos colonos. Estas tierras producían cáñamo silvestre que servía para alimento de ganado, por lo que su explotación se convirtió en un medio de subsistencia para algunos ganaderos de los dos lados de la frontera. Hay en ese tiempo un periodo de hostigamiento que sufrieron los grupos indígenas cucapás, dieguinos y yumas para que desalojaran sus tierras, luego del despojo que ocasionó la guerra provocada por Estados Unidos, que llevó a la pérdida de más de la mitad de nuestro territorio. Con la construcción de vías de comunicación terminó el aislamiento de la región, creció la circulación de mercancías acelerada por la fiebre del oro en California y se dieron fuertes corrientes migratorias hacia estos lugares.

Ya desde fines del siglo xIx, el $80 \%$ de Baja California estaba bajo el control de las compañías deslindadoras y colonizadoras extranjeras. Se iniciaron entonces estudios para aprovechar las aguas del Río Colorado, descubriéndose que entre el río y el Valle Imperial había grandes dunas que, entre otros factores, impedían el paso del canal de riego según la tecnología de la época. Este detalle obligó a bajar el canal por México, introducirlo por el rancho Los Algodones, recorrer 40 kilómetros al oeste y luego reingresarlo a Estados Unidos por donde hoy es Calexico. El gobierno porfirista lo autorizó en 1904 (después de que ya se había construido) poniendo como condición destinar la mitad de las aguas a lo que sería el valle de Mexicali (Estrella, 1980). Anguiano refiere muy bien este proceso al decir que: "Como en otras regiones en las que una agricultura comercial se implanta en tierras vírgenes o poco explotadas, el capitalismo tuvo que transportar consigo las premisas de su funcionamiento" (Anguiano, 1996: 54- 55).

Durante más de 30 años, el VM XI se encontró en manos de compañías transnacionales de capital estadunidense que controlaban los principales medios de producción (tierra, agua e infraestructura) y desarrollaban una agricultura con características de plantación que lograba incorporar tecnología moderna (Stamatis, 1987). ${ }^{2}$ La pro-

2 El control de estas extensiones de tierras lo había obtenido la compañía extranjera mediante la adquisición al posesionario Guillermo Andrade, a quien el gobierno mexicano se las había concesionado en el siglo xıx para que las trabajara y poblara con mexicanos, cosa que nunca cumplió. 
ducción estaba destinada a la exportación y el monocultivo algodonero constituía la característica esencial, con lo que se manifiesta que este valle estuvo desde su inicio ligado al mercado mundial.

Para 1905, la Colorado River Land Company controlaba casi 400 mil hectáreas del valleeimplantó el monocultivo del algodón en 1912 por su excel entecotización en el mercado internacional, conformando lo que se llamó "el rancho algodonero más grande del mundo". Llegaron entonces trabajadores inmigrantes chinos, japoneses ehindúes provenientes del estado de Cal ifornia; a al gunos la compañía les arrendaba mediante contratos de aparcería con el compromiso de sembrar sólo al godón (Garduño, 1992).

Durantelos primeros treinta años del siglo xx secreó un incipiente mercado laboral, conformado por mano de obra migrante de origen asiático y mexicano complementada con al gunos trabajadores indígenas de la región, principalmente cucapah. En la segunda mitad de los años veinte fue importantela reglamentación al trabajo ordenada por A belardo L. Rodríguez, que obligaba a las empresas a emplear un $50 \%$ de trabajadores mexicanos (Moreno, 1994a).

En los últimos años de la década de los treinta, un movimiento social aunado a una política estatal favorable permitió la llegada dela reforma agraria al valle, lo que trajo consigo una nueva configuración en la propiedad dela tierra, mas no en la producción. Las tierras fueron entregadas a los antiguos jornaleros migrantes y campesinos mexicanos que laboraban para las empresas extranjeras bajo las modalidades deejido, pequeña propiedad y colonia, siendo la primerala que se convirtió en la principal forma de tenencia de la tierra en ese tiempo. ${ }^{3}$ Por lo anterior, la estructura agraria del valle de Mexicali sufrió una transformación radical. Ante la nueva situación regional, el capital extranjero no abandonó sus intereses, sino que utilizó nuevas formas para continuar manteniendo su dominio, como la instalación de empresas refaccionadoras que otorgaban créditos e insumos a cambio de las cosechas.

${ }^{3}$ Luego de la entrega de las tierras hubo algunos intentos por desarrollar su explotación colectiva, mismos que no fructificaron. 
Tras la reforma agraria, el gobierno mexicano realizó un fuerte apoyo en infraestructura y tecnología a los nuevos productores, dotándolos de tractores y aditamentos agrícolas nuevos. También los créditos de la banca nacional y el apoyo técnico comenzaron a fluir: se realizó la constitución y rehabilitación del distrito de riego.

U so de agroquímicos durante el periodo

El uso de productos químicos en la agricultura del valle, como explicábamos al princi pio, está íntimamente relacionado con el tipo de desarrollo agrícola que se adoptó, en este caso la vía agroindustrial, que implicaba, entre otros factores, una producción a gran escala de un cultivo que pudiera ser comercializado y tuviera una gran demanda en los mercados internacionales, como el al godón. La fase experimental inició en 1912 (año en que sesembraron 12 hectáreas obteniéndose una cantidad de 15 pacas de 26.5 kilogramos cada una), y a partir de ahí ése fue el cultivo por excelencia.

Durantelos primeros años no existían plagas ni enfermedades que representaran un verdadero peligro para las cosechas, puesto que la producción todavía era incipiente; además, al constituir una zona de reciente incorporación a la explotación agrícola comercial, las condiciones naturales no habían sido gravemente al teradas.

Algunos arbustosy "malas hierbas", como el zacatej ohnson, el trompillo y el zacate bermuda natural, sí llegaban a constituir un problema para las cosechas, pues competían con el algodón por los rayos del sol. Sin embargo, las prácticas de cultivo adecuadas y en los tiempos recomendados evitaban su propagación, por lo que no había necesidad de utilizar productos químicos (DeVivanco, 1924).

No fue sino hasta la década de los años veinte cuando las plagas comenzaron a manifestarse. Según se relata en el trabajo de Sánchez Ramírez (1990), el gusano perforador de la hoja hizo estragos en la producción al godonera dela nacientecooperativa dela Colonia Progreso en 1925, durante el periodo gubernamental de A belardo L. Rodríguez, por lo que se buscaron alternativas químicas. Después aparecieron otras plagas que comenzaron a causar daños más severos a la producción. 
Los productos utilizad os para combatir las nacientes plagas en esos años eran fundamentalmente los venenos queatacaban el aparato digestivo delos insectos, como el arseniato de plomo, el arsénico blanco y el Ilamado verde de París, que se combinaba con azufre y cal. Estos insecticidasinorgánicos, destinados sobretodo al control del gusano bellotero y la chinche lygus - principales plagas del algodonero de ese tiempono presentaban serios daños por acumulación residual en el medio ambiente porque su uso era bastante restringido y además existía un control biológico natural quemantenía a las plagas en niveles controlables (Sánchez, 1990: 238).

La forma de aplicación de estos plaguicidas era manual o con el apoyo de aperos de tracción animal, de modo que los riesgos decontaminación se circunscribían a la zona tratada. Los compuestos inorgánicos eran baratos y fáciles de adquirir en Estados Unidos.

En cuanto al uso defertilizantes, se sabequesólo seaplicaban abonos naturales como el estiércol, ya quelas tierras del vallemexicalense, ricas en limos, no requerían en realidad otros nutrientes, además de que todavía no se hacía un uso intensivo de la tierra.

Al lado del principal cultivo, el algodón, existían otros que sin tener la misma importancia económica se sembraban continuamente, como el trigo, la alfalfa y al gunas hortalizas cultivadas por los arrendatarios chinos, así que estos cultivos también comenzaron a presentar problemas de plagas. En los años cuarenta, la roya del trigo arrasó con la mayor parte de las 40 mil hectáreas sembradas y las pérdidas fueron totales (Sánchez, 1990: 239).

En resumen, los plaguicidas y fertilizantes utilizados desde el inicio de la producción hasta la década de los años cuarenta fueron compuestos inorgánicos y abonos naturales. ${ }^{4}$

\footnotetext{
${ }^{4}$ Las sustancias naturales forman parte del medio ambiente biótico y abiótico y son esenciales para la vida; son permanentes, de baja toxicidad aguda en general y particularmente en mamíferos. Los compuestos inorgánicos -no sintéticos- son permanentes, tóxicos para plantas y animales, especialmente para los invertebrados y vertebrados pequeños, pero de baja toxicidad aguda.
} 


\section{Segundo periodo, 1948-1965: el auge de los plaguicidas organoclorados ${ }^{5}$}

\section{Características del periodo}

El periodo de 1948 a 1960 se considera como una etapa de bonanza para las empresas y los agricultores del valle de Mexicali, pues se llegó a rebasar la cantidad de 100 mil hectáreas sembradas, ${ }^{6}$ manteniéndose constante durante varios años. Había un buen mercado mundial, un tipo de cambio subvaluado y una escasa integración territorial, lo que propició la dependencia regional de los bienes de consumo importados. Varias décadas después de la nacionalización de la tierra, el valle seguía más integrado al mercado de Estados Unidos que al nacional (Stamatis, 1992). Los precios del producto se elevaron hasta diez veces más que los registrados en los años treinta y las ganancias de las empresas refaccionadoras eran cuantiosas.

Varios acontecimientos exógenosy endógenos influyeron en el vaIle para que éste adquiriera importancia en el ámbito mundial, entre ellos la devaluación del peso mexicano quehizo al algodón más competitivo en el mercado mundial, la guerra de Corea que el evó su precio ,y la inversión de capital por parte del sector gubernamental federal que permitió un mejor desarrollo.

En cuanto a la estructura agraria, para este periodo el sector ejidal se afianza como el preponderante, los intentos de cultivar la tierra en forma colectiva fracasan y los agricultores prefieren la parcela individual tipo farmer. El modelo deagricultura industrial basado en monocultivo algodonero continuó.

Es precisamente en esos años cuando se impulsó en el ámbito nacional un modelo de desarrollo que daba prioridad a la industrialización por medio de la sustitución de importaciones y el

${ }^{5}$ Los plaguicidas organoclorados son derivados de los hidrocarburos clorados, son persistentes en el medio ambiente y actúan como venenos estomacales y de contacto. El compuesto más conocido de esta categoría toxicológica es el DDT. Para datos más amplios sobre este tipo de plaguicidas consúltese Albert, 1990.

${ }^{6}$ En el periodo 1955-1956 se alcanzó la cifra de 189 mil hectáreas cultivadas de algodón. 
desarrollo agrícola de alta tecnología, lo que llevó a la adopción de una nueva estrategia "unimodal" en el campo mexicano, en donde el Estado otorgó un fuerte apoyo a la agricultura moderna basada en la irrigación y la producción de cultivos comerciales, y al mismo tiempo, marginó a las explotaciones campesinas. La llamada "revolución verde" hizo su aparición en nuestro país. Este valle, al igual que los demás del noroeste, encajaba a la perfección en el prototipo de agricultura que demandaba esa nueva visión para implementar sus propuestas. Por esa razón se introdujo el paquete tecnológico propuesto por la "revolución verde".7

En las dos décadas que siguieron al reparto agrario en el VMxl, las condiciones de sujeción de los productores por parte del capital extranjero persistieron. El control del agua siguió en manos de una empresa extranjera y los créditos continuaron otorgándolos en su mayoría las empresas refaccionadoras (principalmente de origen estadounidense); éstas tenían plantas despepitadoras, mezcladoras de insecticidas, compresoras, fábricas de manteca y aceite, jabón; además de comisionistas en la venta de maquinaria agrícola, combustibles, fertilizantes, servicios de fumigación, etcétera (García, 1958).

Con su inserción en la división internacional del trabajo, el valle sirvió además como asentamiento "puente" entre los lugares de origen de los trabajadores migratorios del interior y los lugares de destino en zonas agrícolas de Estados Unidos, principalmente Cal ifornia. Se abastecía a su industria textil, se importaban bienes de consumo para la población e insumos y maquinaria y se recibía inversión para financiar la producción algodonera (Stamatis, 1992).

El programa bracero y la gran demanda que había de la fibra en el ámbito mundial intensificaron los flujos migratorios de los estados de occidente y noroeste del país hacia la región. Prácticamente en este periodo fue cuando se experimentaron en la entidad los más grandes promedios de inmigración y crecimiento en toda su historia, con tasas del 9.4\% según M artínez (1987: 21). Esto trajo un cambio en la composición dela fuerza detrabajo, creando un mercado laboral temporalero

7 En realidad esta estrategia era del capital internacional, y los experimentos más eficientes se desarrollaban en México y la India. 
sujeto a los vaivenes de la producción. Durante varios años se rebasó la cantidad de 100 mil hectáreas sembradas, dando trabajo en los cincuenta y sesenta a 30 mil pizcadores temporales, de los cuales cuando menos las tres cuartas partes eran migrantes. A parte de la pizca, la industria al godonera generaba 4 mil empleos obreros deplanta en la actividad de procesamiento del producto en despepitadoras, industrias extractivas deaceite, molinos, desborradoras, fábricas de trapeadores, colchones, etc., además del comercio y servicios financieros ligados al algodón, con lo quehubo un éxodo depoblación rural hacia la ciudad (Moreno, 1994a).

\section{U so de agroquímicos durante el periodo}

En el ámbito mundial, como resultado delas innovaciones tecnológicas generadas en la Segunda Guerra Mundial, se presentó un auge comercial de los plaguicidas organoclorados; Ios valles de Mexicali e Imperial resultaron influenciados rápidamente por ese proceso. Por ese tiempo se inició el uso de plaguicidas sintéticos modernos, ocupando el DDT el primer lugar en cuanto a volumen utilizado.

El desarrollo de una agricultura intensiva y competitiva para el mercado mundial hacía necesario el uso de productos químicos para crecer y combatir las nacientes plagas. El paquete tecnológico de "la revolución verde" traía esas recomendaciones; la justificación ideológica para el uso de plaguicidas y fertilizantes modernos era el aumento de la producción de alimentos para combatir el fantasma de la hambruna. ${ }^{8}$

En pocosaños, el control químico seconvirtió en el principal método utilizado para controlar las plagas. Y también, como decíamos, la

\footnotetext{
${ }^{8}$ El uso del control químico de las plagas se propagó por todo el planeta de una manera vertiginosa a fines de los años cuarenta, como parte de una estrategia dictada por los países industrializados. Algunos autores hacen hincapié en que los plaguicidas, como productos tecnológicos, no son un resultado natural ni tampoco un producto superior en la pretendida evolución unilineal del conocimiento científico y tecnológico dictado por los países más desarrollados; pero sí en cambio son un producto social, resultado de una estrategia tecnológica industrial que se ha impuesto como modelo dominante a escala mundial. Al respecto, véase Bejarano (1989).
} 
vecindad con uno de los valles más ricos de la unión americana, prototipo de la agricultura moderna, contribuyó por el fácil acceso al mercado de plaguicidas modernos.

La instalación de industrias dedicadas a la venta y distribución de agroquímicos se inició precisamente en esa etapa. En 1949 secrea la Compañía Industrial Jabonera del Pacífico (filial de la Anderson Clayton) (Sánchez, 1990). "La Jabonera”, como se le conoció popularmente, estableció un pequeño campo experimental donde realizaban pruebas con semillas de algodonero, uso de fertilizantes y control de plagas. Las recomendaciones que surgían eran inmediatamente aplicadas a través de los inspectores de campo. El abastecimiento de insumos provenía principalmente de Estado Unidos (Sánchez, 1990).

El incremento en los rendimientos de la producción algodonera pronto empezó a manifestarse, sin embargo, las recomendaciones de la "revolución verde" ya comenzaban a tener resultados perniciosos en el valle de Mexicali, al igual que en otros valles modernos.

En los años cincuenta, con el auge en el ámbito nacional y regional de los agroquímicos, algunas empresas extranjeras dedicadas a este rubro instalaron sus filiales en el valle de Mexicali, como Industrias

Cuadro 1. Plaguicidas más utilizados en el valle de Mexicali durante el periodo 1950-1965

\begin{tabular}{lll}
\hline Plaguicidas & Toxicidad & Persistencia en suelos \\
\hline Aldrin & II Alta & Alta (5 años) \\
BHC & III Moderada & Alta (2 a 20 años) \\
DDT & III Moderada & Alta (10.5 años) \\
Dicofol & III Moderada & Alta (2 a 20 años) \\
Dieldrin & II Alta & Alta (7 años) \\
Endrin & III Moderada & Alta (2 a 5 años) \\
Heptacloro & III Moderada & Alta (2 años) \\
Lindano & III Moderada & Alta (2 a 20 años) \\
Metoxiclor & IV Ligera & Alta (18 años) \\
Toxafeno & III Moderada & Alta (20 años) \\
\hline
\end{tabular}

Fuente: Instituto de Investigaciones Sociales, proyecto “Agroquímicos y medio ambiente en el valle de Mexicali", 1995. 
Agrícolas S.A, Química Agrícola del Pacífico, Servicios Técnicos NH3, Insecticidas Ortho, etc. (Sánchez, 1990). La función de las industrias químicas no se circunscribía a la simple venta, sino que también otorgaban servicios de asesoría técnica, recomendando obviamente sus productos para el combate de las diferentes plagas, así como fertilizantes y semillas mejoradas.

El uso de plaguicidas en la agricultura local siguió su paso inexorable, incursionando cada vez nuevos productos, en su gran mayoría compuestos organoclorados. Pero poco a poco las plagas fueron adquiriendo mayor resistencia a los productos químicos y comenzaron a proliferar sin control. ${ }^{9}$

Debido a esta situación se consideró necesario la aplicación de las llamadas "bombas" o cóctel es dediversos compuestos, siendo losmás comunes el DDT más toxafeno y azufre. Estas fórmulas había que aplicarlas en forma aérea para quetuviera mayor efectividad (Miranda-Meneses, 1982: 25). Las “bombas" ocasionaron la destrucción de gran partedela fauna benéfica denominada "predadores" quecontribuía al combate de las plagas, por lo que éstas proliferaron y otras nuevas aparecieron.

Las fumigaciones también comenzaron a producir impactos negativos en los ecosistemas del valle, porque no sólo llegaba el compuesto químico al área cultivada sino también a las poblaciones adyacentes; los plaguicidas tienen una capacidad extraordinaria para viajar hacia todos los recursos naturales. Según Miranda y Meneses (1982), las primeras aplicaciones de este tipo provocaron la muerte inmediata de algunas especies de aves domésticas cerca de los campos de cultivos tratados.

Con el surgimiento de las resistencias en las plagas y el descubrimiento del impacto negativo de estetipo de compuestos químicos en el medio ambiente y la salud humana en el mundo, se buscó otra clase de plaguicidas que no fueran de alta residualidad, como los

\footnotetext{
${ }^{9}$ Las resistencias en las plagas son entendidas como la capacidad de los organismos-plaga para desarrollar líneas genéticas que puedan sobrevivir expuestas a las dosis a las queeran susceptibles las generaciones anteriores. Al respecto consúltese Albert (1990).
} 
organofosforados, introducidos al valle a partir de $1954 .{ }^{10}$ Entre los principales productos seencontraban el $P$ aration Etílico y el $M$ alathion, dealta toxicidad y amplio espectro deacción (Miranda-Meneses, 1982). También se aplicaron al gunos carbamatos como el carbaril. ${ }^{11}$

A mediados de los años sesenta comenzó a ded inar en forma vertiginosa el uso de la mayoría de los organoclorados en los valles de Mexicali e Imperial (Samplin, 1989), situación que coincidió con el principio de una crisis de producción en el valle de Mexicali.

A pesar delas supuestas "bondades" dequevenían precedidos los nuevos compuestos químicos, recién lanzados al mercado mundial por las empresas trasnacionales para sustituir a los anteriores, la utilización de plaguicidas organofosforados no segeneral iza en esos años, pero los productores agrícol as podían adquirirlos mediante las compañías refaccionadoras instaladas en Mexicali.

\section{Tercer periodo, 1965-1980: uso generalizado de plaguicidas organofosforados}

\section{Características del periodo}

Duranteesteperiodo, la producción algodonera en el valledeM exicali entra en una aguda crisis sin retorno. Los precios del producto en el mercado mundial dedinaron considerablemente debido a varios factores, entre los que podemos mencionar la entrada de nuevos competidores al mercado mundial, como China y Rusia, y el surgimiento de las fibras sintéticas. La fuerte ligazón de la economía local, basada fundamentalmente en un producto, con un mercado mundial al cual no podía influir, y mucho menos controlar, trajo consecuencias negativas para la producción del valle.

${ }^{10}$ Los plaguicidas organofosforados son generalmente esteres del ácido fosfórico. Son inhibidores de la enzima acetilcolinesterasa y provocan reacciones en el sistema nervioso. Son poco persistentes en el medio ambiente, pero altamente tóxicos para aves, mamíferos y en menor cantidad para los peces (Albert, 1990).

${ }^{11}$ Los plaguicidas carbamatos tienen algunas propiedades similares a los organofosforados también son inhibidores de la enzima acetilcolinesterasa, pero éstos, derivados del ácido carbámico, son menos persistentes que los organofosforados y sus efectos no duran más de ocho horas. 
Aunado a esto, se presenta un serio problema de salinidad de las tierras del VMxl provocado por las aguas provenientes de Estados Unidos, que también trajo sus repercusiones en la fertilidad de los suelos y por endeen la producción. Y por si fuera poco, nuevas plagas comenzaron a adquirir resistencia y otras, queeran endémicas, seconvirtieron en un problema. Ante tal situación, la inversión extranjera (la Anderson Clayton) decidió abandonar la región.

Debido a la crisis del al godón, el Estado entró al rescate canalizando recursos para la introducción de otros cultivos y provocando la diversificación dela producción; seimpulsó, por ejemplo, un programa ganadero incentivando a los productores de forrajes, granos básicos y cártamo. Las hortal izas y frutales hicieron su aparición en forma masiva y el patrón de cultivos se transformó, perdiendo el algodonero su predominio. La crisis agrícola no fue privativa deesta zona, prácticamente en todo el país se estaba presentando, lo cual denotaba un agotamiento del modelo agrícola dela "revolución verde".

En este periodo encontramos un mercado laboral local ya estable, conformado por trabajadores agrícolas que migraron al valle en los tantos flujos temporales y que se fueron radicando en la localidad; también encontramos a las nuevas generaciones que habían nacido ya en el estado y que continuaban desarrollando las actividades de sus padres. Al reducirsela producción, también se redujo el flujo migratorio. Es a partir de los años setenta cuando crecela inversión estadounidense a través de contratos de producción de hortalizas de exportación, utilizando sobre todo a trabajadores locales, con lo cual aumenta la oferta de empleo durante la mayor parte del año en la agricultura regional.

Luego de la crisis del algodón de los años sesenta, los setenta vieron emerger la producción dehortal izas de exportación queaumentaron la oferta de empleo durante la mayor parte del año y ya no sólo por temporadas. Se inició así la agricultura por contrato, forma de integración de las empresas transnacionales en la producción de hortalizas para procesamiento industrial o consumo fresco de exportación. En esta modalidad, "las empresas suministran créditos, asistencia técnica, insumos y hasta maquinaria agrícola a cambio desu compromiso en ocasiones escrito de entrega de la cosecha por parte del productor" 
(Rama, citada por Stamatis, 1992:10); son pequeñas y medianas compañías de la frontera sur deEstados Unidos distribuidoras de productos frescos.

Estos contratos exigen una demanda efectiva con el evado poder de compra y fuertes inversiones, pues sus costos de producción son más altos por hectárea que los tradicionales (de cuatro a seis veces más queel al godón, deocho a docemás queel trigo), una rápida y eficiente comercialización (la vida perecedera de no más de dos semanas de las hortalizas implica altos riesgos), una zona agrícola propicia con agua disponible, pocas Iluvias eincorporación de recursos técnicos, y agricultores con experiencia productora en cultivos al tamentecomerciales; existe en los contratos una relación directa con el proceso de

Cuadro 2. Plaguicidas más utilizados en 1969 en el valle de Mexicali

\begin{tabular}{ll}
\hline Plaguicidas & Clasificación \\
\hline Azodrin & Organoclorado \\
BHC & Organoclorado \\
Bildrin & Organoclorado \\
Carbicron & Organofosforado \\
DDT & Organoclorado \\
Dieldrin & Organoclorado \\
Dipterex & Organofosforado \\
Endrin & Organoclorado \\
Gusathion & Organofosforado \\
Matahion & Organofosforado \\
Paration Metílico & Organofosforado \\
Perthane & Organoclorado \\
Servin & Organoclorado \\
Thiodan & Organoclorado \\
Toxafeno & Organoclorado \\
Phosdrin & Organofosforado \\
Clordano & Organoclorado \\
Acricid & Nitrofenol \\
Sevin & Organofosforado
\end{tabular}

Fuente: Francisco López Ramos y M. Marroquín, “Intoxicaciones por plaguicidas en Mexicali", Salud pública de M éxico, vol. XIL, número 2, marzo-abril, 1970, p. 200. 
producción. El encarecimiento del agua y del suelo agrícola, junto a los problemas laborales que enfrentó el estado de California en los años setenta, fueron decisivos para que cobrara fuerza aquí la producción de hortalizas. En el valle mexicalense se cultiva cebollín y espárrago.

La gran mayoría de los plaguicidas utilizados coyuntural mente en VMxI para combatir a las plagas eran organofosforados (como los antes mencionados), sin embargo, todavía continuaban predominando los organoclorados, incluso aquel los ya prohibidos en otros países como el Toxafeno y los altamentetóxicos “Drines” y el DDT, ya que se consideraban muy eficientes y de bajo costo. Durante el periodo de 1971 a 1973 se aplicaron en el valle de M exicali 900 toneladas de DDT y formulaciones conteniéndolo (Gutiérrez, 1988), esto a pesar dequesu uso ya estaba en decadencia por su peligrosidad en los países desarrollados. $^{12}$

Con el uso desmedido de plaguicidas se logró controlar a ciertas plagas, pero también se acabó con la mayoría de los predatores. Así, la fauna antes considerada como no dañina para los cultivos, depronto comienza a tornarse en plaga. ${ }^{13}$

A los nuevos cultivos llegaban especies que anteriormente no representaban un peligro, pero bajo las condiciones de ese tiempo se convertían en verdaderas plagas, lo que se debía fundamental mente a practicas erróneas en todo el proceso de la producción y a que se apostaba todo al control químico sin medir las consecuencias. En el

12 Las constantes denuncias de las organizaciones no gubernamentales y acadé micas sobre los efectos crónicos en la salud humana y las devastadoras consecuencias en los ecosistemas a causa de los agroquímicos aplicados, aceleró la sustitución de los organoclorados por plaguicidas organofosforados que, aunque tenían elevada toxicidad y amplio espectro de acción, presentaban poca persistencia en el medio ambiente.

${ }^{13}$ Es durante este periodo cuando al gunos investigadores comenzaron a interesarse por medir el impacto de los compuestos químicos en los ecosistemas y el hombre en la región. Motivados por las voces de alerta que comenzaron a surgir en el ámbito mundial, iniciaron investigaciones para determinar el impacto de los plaguicidas, orgnoclorados, principalmente el DDT. De esta forma, desde la visión de diferentes disciplinas comenzaron a proliferar los estudios. Sin embargo, casi todos se enfocaban al medio ambiente y muy pocos a la salud humana. 
Cuadro 3. Principal es cultivos y las plagas más frecuentes en el valle de Mexicali en la década de los setenta

\begin{tabular}{|c|c|}
\hline Cultivo & Plagas \\
\hline Algodón & $\begin{array}{l}\text { Gusano rosado, gusano bellotero, gusano perforador de la } \\
\text { hoja, fal so medidor, pulga sal tona, chinche, grillo, gusano } \\
\text { peludo, ataña roja, trips, nemátodos }\end{array}$ \\
\hline Trigo & Pulgón \\
\hline Cártamo & Chinche, gusano del girasol \\
\hline Alfalfa & Gusano de la col, gusano picudo, pulgón \\
\hline Sorgo & A raña roja, grillo, gusano soldador, chinche, pulgón \\
\hline
\end{tabular}

Fuente: SARH, Sanidad Vegetal, Guías para producir cultivos en el valle de M exicali, varios años, Mexicali, B.C.

cuadro 3 presentamos las principales plagas que azotaban al valleen los años setenta.

A finales de esos años, con el auge de los plaguicidas organofosforados, los compuestos químicos que anteriormente habían predominado casi desaparecieron. En 1979, en los cultivos al godoneros se aplicaron 680 mil 847 unidades de insecticidas, de las cuales 37 mil 605 correspondieron a productos clorados, es decir, el 5.5\% del total utilizado durante ese periodo. A I año siguiente se acentuó la tendencia a la disminución, que se situó en 1.3\% (Miranda-Meneses, 1982).

Durante el lapso de 1977-1980, el promedio de aplicaciones de insecticidas por ciclo en el algodonero siguió una variación distinta como consecuencia de la importancia otorgada al control natural y al control biológico inducido: de las cinco aplicaciones normales fue disminuyendo hasta tres y media.

De los primeros estudios, el que destaca es el de López-Marroquín (1970), quien analizó la intoxicación por plaguicidas de 59 personas en el $\mathrm{VMxl}$, incluyendo la defunción de dos pilotos fumigadores y dos bandereros que carecían de equipo protector.

En el segundo estudio, de Hernández (1976), se analizaron concentraciones de DDT en organismos acuáticos como el bocón y la lisa, peces en los que se encontraron grandes concentraciones de esta clase de plaguicidas. 
En cuanto a las empresas que el aboraban o distribuían plaguicidas, sabemos que existían nueve formuladoras, almacenadoras y distribuidoras que también realizaban aplicaciones; otras nueve eran sólo almacenadoras, y tres se dedicaban únicamente a realizar aplicaciones en el medio agrícola.

También había otras diez empresas que realizaban fumigaciones aéreas como: Empresas N aul, Fumigadora García, Fumigadora Canton, Servicio NH3, A gricultores Industriales, CAPSA, Banco Ejidal, Fumigadora El Capitán y Fumigaciones Aéreas del N oroeste. Las compañías trabajaban eventual mente sólo por temporadas y se abastecían de las demás empresas almacenadoras. ${ }^{14}$

En el medio urbano se contaba con cuatro fumigadoras dedicadas al control dela fauna nociva en las zonas comerciales y residenciales; en estas últimas no se realizaban formulaciones ni mezclas.

\section{Cuarto periodo: de los años ochenta hasta la actualidad}

Características del periodo

En esta etapa el VMxl entra en las nuevas tendencias de reestructuración productiva y modelosflexibles quecomenzaron a implementarse en el ámbito mundial. La necesidad de lograr mayor competitividad en el mercado internacional mediante estrategias que permitieran diversificar la producción y mejorar la calidad y presentación de los productos obligó a los productores a cambiar sus conceptos y prácticas agronómicas para insertarse en las reglas que estaba imponiendo el mercado.

Sólo aquellos capaces de tener las condiciones adecuadas para la competencia podrían tener futuro, y para ello había que buscar nichos de cultivos que sirvieran como complemento de la demanda de productos no abastecida en los mercados. Ésa era la nueva ola empresarial que se pregonaba.

14 También en 1975 algunos investigadores realizaron estudios sobre concentraciones de DDT en almejas y sedimentos de todos los canales del valle, encontrando grandes cantidades del compuesto y sus metabólicos. 
Las nuevas políticas agrarias nacionales venían precedidas también de esta nueva visión eficientista, así que la desincorporación de las empresas estatales que brindaban apoyo al campo, el retiro de los subsidios, el fin de los precios de garantía, la imposibilidad de créditos y el cambio en las leyes agrarias que permitieron la renta y venta de la tierra, terminaron por afectar a los productores de los cultivos tradicionales.

De esta forma seobserva en los años ochenta el auge de la agromaquila, la agricultura por contrato y los enclaves de exportación del cebollín y otras hortalizas. También se da una crisis sin retorno de los cultivos tradicionales, como el algodón y el trigo.

\section{La composición de la fuerza de trabajo asal ariada \\ en el mercado laboral de las hortalizas}

El auge delos cultivos deexportación modificó el patrón deacumulación capitalista en la agricultura tendiente a la industrialización y amplió la fuerza de trabajo asalariada, lo que trajo consigo “ modificaciones en la composición y comportamiento de la fuerza de trabajo asalariada en relación a la estructura de edad y sexo, especialización de la fuerza de trabajo, corrientes migratorias y salarios" (Barrón, 1992: 1). Se usa intensivamente la mano de obra e insumos más industrial izados externos a los ecosistemas agrícolas, con aprovechamiento más eficiente del agua, la siembra de monocultivos y, sobre todo, una utilización extraordinaria de insumos químicos que permiten una mayor fertilidad de la tierra (Bejarano, 1989).

Los procesos de reestructuración y de movilidad del trabajo -determinados por la flexibilización y desregulación laboral- están remodelando profundamentea las sociedades rural es que participan en las dinámicas económicas globales en la manera en que las familias jornaleras resuelven su reproducción.

Los criterios de calidad y productividad a nivel internacional se colocan cada vez más por sobre los criterios de uso y valoración dela fuerza de trabajo en nuestro país, aumentando la vulnerabilidad de las familias jornaleras y, eventualmente, posicionándolas en situaciones de mayor riesgo social, económico y de salud para las siguientes 
generaciones. Hoy en día, la agroindustria está desarrollando procesos de trabajo flexibles que incluyen mano de obra femenina e infantil y la precarización del trabajo que agudiza aún más sus condiciones de explotación (Barrón, 2002). Esto ha llevado a un mayor deterioro de las condiciones de reproducción social de las familias jornaleras que laboran en el valle de Mexicali, lo que las obliga, en aras de la supervivencia, a incorporar al mercado laboral a sus niños y niñas (López, 1998).

Mientras que en los mercados de trabajo secundarios o menos desarrollados predomina la mano de obra masculina, en los primarios o desarrollados (hortalizas, frutas de ciclo corto y flores) hay una tendencia a la predominancia femenina einfantil. Hay así regiones donde las mujeres adultas forman el $50 \%$ del mercado de trabajo, y niños y niñas de 14 años o menores, el 20\%. Estos mercados colocan grandes volúmenes de producción en los mercados de exportación, y conforman una marcada división social del trabajo y, por tanto, una demanda mayor de fuerza de trabajo especializada (Barrón, 1992). Al lado dela feminización del trabajo agrícola ocurre cada vez más la infantilización del mismo, ya que las mujeres son acompañadas frecuentemente por sus hijos, que ingresan prematuramente al trabajo.

Datos oficiales consideran que las exportaciones hortícolas en el estado le siguen en importancia a las maquiladoras. En el cebollín, el mercado potencial estadounidense es de 74578796 dólares. En Mexicali, en los ciclos otoño-invierno 1995 y primavera-verano 1996, la superficie sembrada de cebollín fue de 2776 hectáreas, con una producción de 34042 toneladas y un valor de 195419000 pesos, y en los mismos ciclos de 2000 y 2001 fue de 4457 has, se produjeron 55161 toneladas con un valor de 312669000 pesos según la Secretaría de Fomento A grícola del Estado de Baja Cal ifornia (2004).

Contrastan con este crecimiento los problemas en otros cultivos regionales: créditos insuficientes e inoportunos, caída de los precios internacionales del algodón y de los precios de garantía aquí, especial mente del trigo, contracción dela demanda forrajera-pastos-, contaminación salina por falta de rehabilitación de las tierras, grave 
deterioro del sistema de riego, ineficiencia del uso del agua y reducción por desaparición de excedentes del Río Colorado existentes desde los setenta (Díaz Hermosillo, 1995).

La desmesurada diferencia sal arial (6.50 dólares la hora en Estados Unidos y al rededor de 50 centavos de dólar en México), la disponibilidad del agua y dela tierra, así como el bajo costo deesta última, son factores decisivos para atraer los cultivos hortícolas a la localidad. Actualmente, entre el 70 y el $80 \%$ de las parcelas, en ocasiones ejidos enteros, están rentados. ${ }^{15}$

Hoy en día, por un lado encontramos un valle agrícola pobre, en crisis, con problemas de comercialización de los cultivos tradicionales, hasta con desempleo que repercute fundamental mente en el sector ejidal y de la pequeña propiedad; pero por el otro, tenemos un valle agrícola rico, con especialización en algunos cultivos hortícolas quetienen una gran demanda en los mercados internacionales, y que beneficia a unos cuantos productores. Se da una especialización en la producción al imentaria de exportación, y aunque sea poca la superficie cultivada, es muy importante el valor de la producción y del empleo. Por ejemplo, en 1986 y 1987 era sólo el 4.5\% de la superficie, pero el $19.5 \%$ del val or y el $33.85 \%$ del empleo sin considerar el empaque de la producción (Stamatis, 1992); en años más recientes, se ocupa al rededor del $10 \%$ de la tierra cultivable.

\section{U so de plaguicidas en este periodo}

La etapa que abarca desde los años ochenta hasta la actualidad se caracteriza por el retorno de las plagas y el uso de una gran diversidad de agroquímicos, desde organoclorados, organofosforados y carbamatos hasta los de la tercera generación: piretrinas, piretroides y plaguicidas ecológicos. En 1981, del total de plaguicidas aplicados al cultivo del al godonero sólo 1.27\% correspondió a organoclorados, entre los cuales ya no figuraba el DDT (Nieblas-Ortiz, 1986).

${ }^{15}$ Por ejemplo, en 1994 en el Ejido Cucapah Mestizo, de 65 parcelas, 52 estaban rentadas y abundan los embargos a ejidatarios (García, 1994). 
Cambiaron las condiciones y los modelos del desarrollo agrícola, pero las prácticas agronómicas no mucho. Se continuó dando prioridad al control químico sobre otro tipo decontroles como el biológico, integrado y natural. En determinados momentos, cuando las plagas arreciaban y no había posibilidad de erradicarlas, se le dio importancia a otro tipo de controles.

En este periodo se da una gran difusión a las al ternativas al uso de plaguicidas sintéticos. La conciencia de los graves daños a los ecosistemas, y al organismo humano en especial, obliga a los países a tomar medidas mucho más drásticas para controlar el uso de los químicos con mayor riesgo decontaminar los al imentos y la salud humana. Los plaguicidas siguieron siendo motivo deinterés especial mente para estudiantes de química y agronomía, por eso se presentan al gunos trabajos de tesis encaminados a esta temática. ${ }^{16}$ También al gunos investigadores de la Universidad Autónoma de Baja California realizaron trabajos para medir la incidencia de organoclorados (Gutiérrez, 1988), sin embargo, no se hizo ningún estudio sobre la salud humana.

La preocupación de los productores mexicalenses, especial mente delos hortícolas, por las posibilidades de que sus productos exportables fueran devueltos por contener residuos de plaguicidas se materializan en políticas de inocuidad. El uso de compuestos con menor persistencia y residualidad es la política actual.

A pesar de que la tendencia hacia la disminución del uso de los plaguicidas organoclorados era evidente en la década de los ochentas, sí continuaron utilizándose al gunos de estos productos en cantidades pequeñas, en forma encubierta o mezclados con otros compuestos. Tal es el caso del Endrin, que a pesar de haber sido prohibido por las autoridades sanitarias continuó utilizándose hasta 1985, o el Endosulfan que todavía se aplicaba en el año 2000.

Para 1981, en el al godonero se incrementó de tres a cuatro la cantidad de aplicaciones de plaguicidas; los entomólogos lo atribuyeron

16 Tal es el caso de Carpio (1983), quien estudió al pentacloronitrobenceno (PCNB) en suelos del valle de Mexicali, y Macedo (1987), que se abocó a identificar residuos del organoclorado Endrin (multitox) en aguas de drenes y canales del valle de Mexicali. 
a que en ese año se dieron condiciones adversas a la fibra por una incidencia de plagas no esperada. La realidad es que partir deentonces se presentó un aumento notable en las poblaciones de las principales plagas de este cultivo, así como de otras que anteriormente eran consideradas de carácter secundario, como la mosquita blanca, la araña roja y el gusano perforador de la hoja (Miranda-Meneses, 1982). Lo mismo sucedió con los otros cultivos de invierno tradicionales.

Aunado a esto, también las conocidas como malas hierbas se constituyeron en un problema, sobre todo en cultivos de verano-otoño. Por esas razones, el consumo de plaguicidas siguió creciendo, manteniéndose estable durante el segundo quinquenio de los años ochenta.

El algodón, principal consumidor durante la década de 1980-1990, consumió alrededor del $70 \%$ de los plaguicidas utilizados. Únicamente en insecticidas se aplicaron más de cinco millones de litros, con promedios anuales mayores a 500 mil litros (Moreno, 1994c).

La proliferación de nuevas plagas y las resistencias adquiridas de las que ya estaban trajeron la incorporación de un sinnúmero de productos químicos. Se probaron algunos que tenían baja persistencia y poca residualidad como las piretras y piretrinas, insecticidas sintéticos derivados del crisantemo.

En los noventa, también el uso de fertilizantes sevolvió extraordinario: se vertieron más de medio millón de litros anuales en los diferentes cultivos del valle de Mexicali (SARH, 1992).

Para el año 2000 resultó difícil cuantificar la cantidad de químicos vertidos, sobre todo porque en las hortalizas existen serias dificultades

Cuadro 4. Volumen de insecticidas utilizados en los cultivos tradicionales, Valle de Mexicali, ciclo 1999-2000

\begin{tabular}{lc}
\hline Cultivos & Volumen (lts) miles \\
\hline Algodón & 93821 \\
Trigo & 90817 \\
Alfalfa & 210799 \\
Total & 395435 \\
\hline
\end{tabular}

Fuente: El Colef-Universidad Estatal de Michigan, proyecto "Riesgo ambiental en la salud por el uso de plaguicidas en el valle de Mexicali", 2002. 
para tener acceso a los datos, ya que intervienen criterios de protección al productor por las posibles barreras fitosanitarias para la exportación. De los pocos registros en los cultivos tradicionales se sabe que se utilizaron cerca de 400 mil litros de insecticidas en el ciclo 1999-2000.

Plagas y plaguicidas en el cultivo de las hortalizas

A tención especial durante esta etapa, merecen los cultivos hortofrutícolas por la importancia que han adquirido. Como señalábamos anteriormente, las hectáreas sembradas deestos productos tienen un peso relevante, más que por la superficie, por la capacidad tecnológica y la importancia económica. Con el auge hortícola semultiplica la utilización de herbicidas y funguicidas, que no sólo se utilizan para atacar las enfermedades producidas por hongos o bacterias, sino además de manera preventiva.

Durante los años ochenta, al igual que el cultivo algodonero, algunos frutales sufrieron los estragos de la mosquita blanca, en especial el melón y la sandía. En 1981 esta plaga destruyó más del 95\% de la superficie cultivada de este último producto (Rocha, 1992). La mosquita ya existía en la zona desde varios años atrás, pero hasta 1977 comenzó a tener importancia. En el ciclo deinvierno de 1991, el insecto provocó daños considerables en los cultivos mencionados,

Cuadro 5. Productos utilizados durante los ciclos 1999-2000 y 2001-2002 en los cultivos hortícolas del valle de Mexicali

\begin{tabular}{llll}
\hline Cultivo & Insecticidas & Herbicidas & Fungicidas \\
\hline Gaytan & Succest & Trifluralina, Galigan, Sethoxidin & Ridomil \\
Kale & Succest & Trifluralina, Galigan, Sethoxidin & Ridomil, Bravo \\
Cilantro & Succest & Trifluralina, Galigan, Sethoxidin & - -- \\
Perejil & Succest & Trifluralina, Galigan, Sethoxidin & Ridomil, Bravo \\
Rapini & Succest & Trifluralina & - \\
Leek & Succest, Lannate & Trifluralina, Galigan, Sethoxidin & Ridomil, Bravo \\
Espárrago & Bacilus Tridingencial & Seancor & Bayleton, Tit \\
Espinaca & Succest & Ro-neet, Poast & Ridomil, Bravo \\
Cebollín & Succest, Lannate & Trifluralina, Galigan, Sethoxidin & Ridomil, Bravo \\
Rábano & Pirimor & Trifluralina & Ridomil \\
\hline
\end{tabular}

Fuente: El Colef-Universidad Estatal de Michigan, 2002. 
valuados en 27 mil 500 y 3 mil 825 millones de pesos respectivamente (SARH, 1992).

Entre las múltiples causas que originaron la infestación de la mosquita blanca en estos años, se señal an como los más importantes el desarrollo deuna al ta resistencia a insecticidas y la eliminación desus enemigos naturales, como parásitos y predatores, por prácticas agroeconómicas inadecuadas. Los cultivos preferidos por la mosquita blanca eran melón, sandía, calabacita, pepino, rapini , lechuga, coliflor, tomate, vid, cítricos y al godón (SARH, 1992).

Por otro lado, las hortalizas son los cultivos que demandan fuertes cantidades de fertilizantes, pudiéndose aplicar hasta 400 kilos de nitrógeno por hectárea, y como en el valle se haceun uso intensivo delas tierras, acostumbran aplicar varias veces durante el ciclo de cultivo. Algunos de los productos hortícolas más importantes gastan un al to porcentajeen productos químicos. En la actual idad podemosencontrar un gran número de plaguicidas, como puede verse en el cuadro 6 .

Lo que se deduce de aquí es que los productos utilizados por los agricultores dehortal izas, en especial por los quedestinan su producción para la exportación, no son los que están restringidos o prohibidos por la Agencia de Control Ambiental del gobierno de Estados Unidos (EPA, por sus siglas en inglés). Seguramente ellos sí seapegan a las disposiciones internacionales, sobre todo a las de esta instancia, y no seexponen al rechazo desus exportaciones. Los plaguicidas permitidos por ella son de persistencia ligera en el producto, como los organofosforados, carbamatos y piretroides.

\section{U so de agroquímicos, contaminación ambiental y salud pública}

En todo estepanorama del uso deagroquímicosen el valledeM exicali, conviene hacer una acotación importante en lo referente a la salud pública y la contaminación del medio ambiente.

Al revisar la literatura médica sobrecontaminación producida por agroquímicos en humanos, nos encontramos con una investigación realizada por el Instituto Tecnológico deSonora en la zona dela Costa de Hermosillo, en el vecino estado donde, al igual que en el valle de Mexicali, abundan los cultivos de exportación. Los resultados mos- 
Cuadro 6. Principales agroquímicos utilizados en todos los cultivos hortícolas del valle de Mexicali en 2003-2004

\begin{tabular}{|c|c|c|c|}
\hline Insecticidas & Herbicidas & Fungicidas & Fertilizantes \\
\hline Rotor & Iloxan & Bayleton & Urea \\
\hline Tamaron & Bromilan & Rydomil & Fosfato \\
\hline Supracid & Treflan & Saprol & Amoniaco \\
\hline Gusathion & Gesapriv & Tilt & F. Foliar \\
\hline Hostation & Poast & Manzate & Nitrofos \\
\hline Cymbush & Eptam & Nemacur & $\mathrm{NH} 3$ \\
\hline Thiodan & Faena & Topik & Triple 15 \\
\hline Lannate & Puma & Bravo & $\begin{array}{l}\text { Fosfato } \\
\text { Monoamoniaco }\end{array}$ \\
\hline Lorsban & Seancor 7 & & UAN-32 \\
\hline Malathion & Dactal & & \\
\hline Sevin 80 & Topyk & & \\
\hline Phosdrin & Grasp & & \\
\hline Folimat & Butyrac & & \\
\hline Trigard & Gramoxo & & \\
\hline Endosulfan & Defolia & & \\
\hline Diazinon & Trifluralina & & \\
\hline Temik & Sethoxidin & & \\
\hline Cytrolane & Galigan & & \\
\hline Dippel & Bacilus & & \\
\hline & Tridingencial & & \\
\hline Metamidofos & Poast & & \\
\hline Gusathion Metílico & & & \\
\hline Dimetoato & & & \\
\hline Herald & & & \\
\hline Orthone & & & \\
\hline Asifos Metílico & & & \\
\hline Bromuro De M etilo & & & \\
\hline Naled & & & \\
\hline Paracuat & & & \\
\hline Clorpicrina & & & \\
\hline Azodrin & & & \\
\hline Succest & & & \\
\hline Pirimor & & & \\
\hline
\end{tabular}


traron la presencia de residuos de agroquímicos en la tierra, el agua, en organismos vegetales y animales y en la leche materna de mujeres jornaleras (Moreno, 02/ 07/ 93). Después, en entrevista con especialistas del ramo y estudios de Lilia Albert (1990), nos enteramos de que en el Valledel Yaqui, importantecentro productor agrícola sonorense, médicos del Instituto M exicano del Seguro Social deCiudad Obregón encontraron agroquímicos en la sangre del cordón umbilical de bebés hijos de jornaleros, detectándose por ese tiempo en la región una alta incidencia de leucemia en la población infantil rural. Investigadores del Centro delnvestigación Científica y Estudios Superiores de Ensenada y de Pediatría del ISSSTE en la misma ciudad bajacal iforniana, al investigar casos de mujeres que procrearon hijos con gastrosquisis, ${ }^{17}$ encontraron residuos de pesticidas y bifenilos policlorados (BPC's) en su tejido adiposo, que se transfiere a la leche materna. Ellos señalan que el lindano, un insecticida organoclorado, produce efectos agudos (hiperestesia y parestesia en cara, es decir, espasmos musculares, convulsiones, vértigo, temblor y dificultad respiratoria) y efectos crónicos (cirrosis y hepatitis crónica; afecta la fertilidad masculina porque atrofia los testículos, ocasionando disminución en su peso y en la producción de esperma en ratas). Al lindano tambien se le ha asociado a la anemia blástica y al cáncer cerebral. Estudios recientes (1995) reportados por la Cancer Prevention Coalition de Estados Unidos asocian el uso dechampús de lindano con el cáncer cerebral deniños. En animales es un carcinógeno comprobado y se sabe que causa daño al sistema nervioso de humanos y animales, entre otros graves efectos sobre el sistema reproductivo y endocrino (Valdés et al., 2002).

Un estudio del Centro de Educación sobre Pesticidas deSan Francisco, California, explica que algunos pesticidas (aunque no se puedan ver ni oler) entran al cuerpo por medio de la piel y al aspirarlos o tragarlos (Moses, 1992). Investigaciones realizadas en California han determinado que el riesgo de fallecimiento del feto aumenta $120 \%$ en mujeres que viven cerca de campos agrícolas, existiendo mayor riesgo de muerte fetal por defectos congénitos cuando las

17 Defecto congénito en el cual se produce una abertura en la pared abdominal y las vísceras aparecen expuestas sin ninguna membrana protectora. 
mujeres se exponen a pesticidas en la tercera y la octava semana de embarazo (Valdez et al., 2002). En ciertos países del norte de Europa, investigadores descubrieron en trabajadores expuestos a productos químicos mutaciones en los espermatozoides, como escasez severa y carencia de cola, entre otras (Peña, 1993).

En suma, en la literatura médica se han establecido relaciones de plaguicidas, herbicidas, fungicidas y otro tipo de químicos con problemas demutagénesis, enfermedades crónicas como leucemias, alergias, defectos de nacimiento, alteración al sistema central nervioso y el hígado; además también están relacionados con esterilidad, abortos espontáneos, defectos de nacimiento y muerte del feto (Moses, 1992), independientemente dela generación deagroquímicos a la que pertenezcan, así sean organoclorados, organofosforados, carbamatos eincluso piretroides.

En el trabajo rural, según estudios de M oses (1992), la principal vía de penetración en el ser humano es a través de la piel; si la piel está mojada o si se suda, entran con mayor facilidad quesi estuviera seca, lo mismo que cuando existen ronchas, escoriaciones y heridas. Setragan residuos de pesticidas cuando se comeen el campo y si seingiere el producto que se está cosechando; se aspiran en el airey son arrastrados por los vientos, lo cual puede provocar una intoxicación aguda. ${ }^{18} \mathrm{Al}$ penetrar en el cuerpo, los plaguicidas pueden llegar hasta la sangre, orina, sudor, saliva, lágrimas, grasa y a otros tejidos y fluidos del cuerpo, entre ellos la leche materna. Al producirse la toxicidad crónica por la exposición frecuente o permanente, los efectos en el organismo tardan tiempo en manifestarse y están relacionados con ciertas enfermedades.

Según los distintos estudios sobre el tema (Albert, 1990; Moses, 1992, Bejarano, 1989), existe una gran indefensión de la población rural, especialmente los más pobres, ante el grado de contaminación provocado por el uso prolongado de los agroquímicos. Las fumigaciones por aire expanden el químico y contaminan agua, aire, suelo y, por supuesto, seres humanos. Lo anterior, aunado a la casi

${ }^{18}$ Los efectos de toxicidad aguda son el envenenamiento y la muerte y afectan todo el organismo. Los síntomas son: dolor de cabeza, mareos, debilidad y fatiga, 
nula vigilancia de las autoridades, sobre todo en el destino de los recipientes, agrava la situación de los habitantes del campo, sobre todo jornaleros. En el valle de Mexicali, por ejemplo, es una práctica cotidiana en las familias jornaleras lavar (para utilizarlos) recipientes de agroquímicos en drenes y canales, donde se bañan niñas, niños y adultos y donde lavan su ropa y utensilios caseros. A pesar de que los recipientes y envases vacíos están confinados en vertederos con cercos de mallas y cemento, no dejan de constituir un riesgo por las Iluvias y el viento; además, existen numerosas pistas de empresas fumigadoras cuyos depósitos resultan insuficientes para al bergar los desechos, ya que no existen un confinamiento mayor autorizado por las autoridades sanitarias.

El desinterés y la situación económica precaria deal gunos productores, en su afán de reducir costos, ocasiona la falta de capacitación para el manejo de pesticidas de sus trabajadores; esto, aunado a la pobreza existente en las zonas de cultivo donde persisten los bajos niveles de escolaridad de los jornaleros, agrava la situación al desconocer el manejo adecuado de sustancias tóxicas.

En el valle de M exicali, el riesgo del contacto también se encuentra en la población deescasos recursos, y en especial en los queviven en las Ilamadas choriceras, áreas de viviendas precarias sin servicios básicos, quienes hacen uso del agua delos drenes o bien en ocasiones pescan en el sistema hidráulico del valle de Mexicali (Moreno, 1994b). Más posibilidades de contaminación se presenta en quienes padecen desnutrición y parasitosis.

Una gran cantidad de plaguicidas prohibidos, restringidos o eliminados en los Estados Unidos por su nivel de toxicidad y daño a la salud humana, y no recomendados por autoridades como la EPA, siguen utilizándose en el valle de M exicali, especialmente en los cultivos tradicionales, entre ellos:

náuseas, vómito y diarrea, sudor excesivo, vista nublada, aumento de secreción de ojos, nariz y boca, dolor en el pecho con dificultad para respirar, calambres y dolor muscular. Si el envenenamiento es severo, pueden presentarse convulsiones, pérdida del conocimiento, entrada de fluido a los pulmones y, de no ser atendido adecuadamente, la muerte. 
- El endosulfan: plaguicida organoclorado perteneciente al grupo de los ciclodinos, comúnmente aplicado para el control de las plagas en los cultivos algodoneros de la región.

- El herbicida Fenoxi 2,4-d: restringido en varios países, se utiliza en Mexicali. Tiene una variedad de presentaciones, pero todas con el mismo ingrediente activo.

- El azinfos metílico: insecticida organofosforado extremadamente tóxico (LDs50 oral), es neurotóxico y en sus efectos ambientales reduce la vida silvestre.

- El metamidofos: insecticida y acaricida organosfosforado, también al tamente tóxico (LSD 50), es fatal si seinhala o es absorbido por la piel; provoca efectos residuales en aves, es tóxico para peces y causa la muerte en el ganado que ingiere el forraje contaminado.

- El paracuat: utilizado en al gunas hortal izas del valle, está señalado en la campaña "La docena trágica" como un compuesto sumamente peligroso y causante de cientos de muertes. ${ }^{19}$

Aun cuando los productores hortícolas han cuidado el uso de plaguicidas restringidos o prohibidos por la EPA ya quela mayor parte dela exportación desus productos se destina hacia Estados Unidos, y a pesar de quehan estado atendiendo las medidas de inocuidad recomendadas por los mercados internacional es, resulta primordial investigar a fondo la magnitud de los daños en la salud de las niñas y los niños, lo mismo que en los adultos y en general en la población rural y urbana del valle de Mexicali, lo que consideramos como un asunto de salud pública que urge atender.

\section{Consideraciones finales}

En la dimensión agronómica, el uso de agroquímicos en el valle de Mexicali ha pasado por un proceso histórico similar al de varias regiones de la República mexicana donde están enclavados distritos

${ }^{19}$ Campaña que realizan las organizaciones civiles internacionales contra 12 compuestos que se ha comprobado causan daños a la salud humana. 
de riego. El modelo agrícola adoptado desde la "revolución verde" ha seguido implementándose, y la dependencia de los insumos químicos ha continuado sin que parezca tener fin.

La justificación del uso de plaguicidas sintéticos fuequecon ellos se lograría elevar la producción y controlar las plagas para dar satisfacción a los requerimientos de una población en aumento, cosa queno ha ocurrido, y los resultados no son nada al entadores. El valledeM exicali actualmente pasa por una crisis económica importante; las plagas tradicionales no han podido ser erradicadas, al contrario, han surgido otras con mayor poder destructivo. Ante esto cabría cuestionarse si vale la pena continuar con un modelo de desarrollo que ha demostrado ser tan depredador para el medio ambiente y la salud humana.

Tal vez habría que explorar otras alternativas aun dentro del contexto actual neoliberal, como el modelo de agricultura orgánica que, aunque presenta propuestas que parecerían utópicas para las condiciones del valle de M exicali, resulta una esperanza para evitar el proceso de contaminación de la natural eza y por ende de la vida misma. Es decir, atender la demanda de la sociedad desu derecho a la salud y a un ambientelimpio y libre de la contaminación.

En la dimensión económica, las políticas comerciales internacionales se orientan a la firma de tratados como el que está en vigor entre México, Estados Unidos y Canadá, que por lo general afectan severamente el desarrollo de los países. En el terreno que nos ocupa, la investigadora Martha Stamatis (15/03/92) advertía a principios de los noventa al go quela realidad actual confirma:

El cultivo de hortalizas, al extenderse (por los bajos costos de la producción aquí, por las reformas al artículo 27, por el Tratado de Libre Comercio) traerá consigo una mayor concentración en el uso deagroquímicos, lo queagudizaráel impacto ambiental y en los trabajadores agrícolas. Si bien es cierto que, siguiendo las disposiciones de la A gencia de Control Ambiental de Estados Unidos, los exportadores de hortalizas están controlando efectivamenteel uso depesticidas en cuanto a que no afecte la seguridad del consumidor, lo es también que no hay ningún control sobre su impacto en las zonas productoras, lo que está provocando fuertes problemas de 
contaminación en la tierra y el agua, y sobre todo en la salud de los trabajadores agrícolas y la población rural. Ambos problemas se agravarán si no se toman desde ahora medidas serias para reducirlos.

Estas medidas incluyen la difusión entre la población de los riesgos del uso de los agroquímicos y las indicaciones para su manejo como una obligación de los productores y las autoridades del medio ambiente, la salud y el trabajo; la inspección rigurosa de los productos que se utilizan en el valle, impidiendo la entrada de los que están prohibidos en Estados Unidos y otros lugares; y la investigación y fomento de procesos y productos deorigen natural necesarios para la agricultura que eliminen el uso de los agroquímicos actuales.

En cuanto a la relación entreel uso deplaguicidas y la salud pública, como señalamos antes, los estudios destinados para medir sus impactos son escasos, la mayoría son de carácter técnico y enfocados a organismos acuáticos, deahí la necesi dad de realizar trabajoscientíficosque seadentren en la salud pública. El aporteque puedan dar las diferentes disciplinas no sólo es valioso sino también indispensable. Con este fin, en la actualidad está en proceso una investigación interdisciplinaria a cargo del Instituto de Investigaciones Sociales dela uABC y del Departamento deSalud Pública de la Facultad de Medicina dela unAm, con la colaboración de la Facultad de Medicina de la UABC, que generará conocimiento -entre otros aspectos- acerca de algunos efectos de los agroquímicos en la salud.

\section{Bibliografía}

Albert, Lilia (coord.) (1990), Los plaguicidas, el medio ambientey la salud, México, Centro de Ecodesarrollo.

Barrón, A ntonieta (1992), Los mercados de trabajo rurales. El caso de las hortalizas en M éxico, tesis de doctorado, Facultad de Economía, UNAM.

et al. (2002) A pertura económica de las frutas y hortal izas de exportación en M éxico. U $n$ acercamiento al estudio de la segmentación de los mercados defuerza detrabajo, Universidad Autónoma de Nayarit. 
Bejarano, Femando (1989), "Transnacionales y uso de plaguicidas", revista Textual, nueva época, número 25 , vol. 2, UACH, junio.

Caldera Brant, Vinicio (1996), citado por María Eugenia Anguiano

Téllez en A gricultura y migración en el valledeM exicali, El Colegio dela Frontera Norte.

Carpio Obeso, María de la Paz (1983), D eterminación de residuos de pentacloronitrobenceno ( $P C N B$ ) en suelos por cromatografía de gases en el valle de M exicali, B.C., tesis, Escuela de Ciencias Químicas, UABC.

Cicoplafest (1991), Catálogo 0 ficial de Plaguicidas.

DeVivanco, Aurelio (1924), Baja California al día. Lower California up to date, Los Ángeles, Ca., Wolfert Printing Co. Edición bilingüe.

Díaz Hermosillo, J. Samuel (coord.) (1995), “Baja California hacia la competitividad Perspectivas de desarrollo para el siglo XXI". Competitividad sectorial, mayo, Mexicali, B.C.

El Colef-Universidad Estatal de Michigan (2002), Proyecto "Riesgo ambiental en la salud por el uso de plaguicidas en el Valle de Mexicali".

Estrella, Gabriel (1980), El origen de la región de los valles de M exicali e Imperial desde la perspectiva de las relaciones sociales, Cuaderno de Ciencias Sociales núm. 1, Instituto de Investigaciones Sociales dela UABC.

García, Max (1958). "El crédito agrícola en el valle de Mexicali", Ponencia presentada anteel Consejo Estatal dePoblación. Mexicali. Garduño, Everardo (1992), V oces y ecos de un desierto fértil, Mexicali, B.C., UABC.

Guardado Puentes, J. (1976), “Concentración deDDT y susmetabólicos en el valledeM exicali y el Alto Golfo deCalifornia", Cooperation 0 cean Fish, Invest., número 18.

Gutierrez Galindo, Efraín et. al. (1988), “Hidrocarburos clorados en moluscos del valle de Mexicali y alto Golfo de California", revista Ciencias $\mathrm{M}$ arinas, número 14 , abril.

Hernández, J.R. (1976), A plicación decromatografía de gases en la determinación de residuos tóxicos de DDT en peces de lagos y canales de M exicali, B.C, México, ESQUIE-IPN . 
López Limón, Mercedes Gema (1998), El trabajo infantil, fruto amargo del capital, edición de la autora, Imprenta San Andrés, M exicali, B.C.

Lopez Ramos, Francisco y Marroquín (1970), “Intoxicaciones por plaguicidas en Mexicali", revista Salud Pública de M éxico, vol. XIL, número 2, marzo-abril, p. 200.

Miranda Meneses, Víctor Manuel (1982), Impacto ecológico por el uso de insecticidas en el valle de M exicali, B.C., tesis profesional, Escuela de Ciencias Agrícolas, UABC.

Macedo, Tones, José Alejandro (1987), Evaluación de residuos tóxicos de endrin (multitox) por cromatografía de gases, en aguas de drenes y canales del valledeM exicali, Baja California, tesis, Facultad deCiencias Agrícolas, UABC.

Mazon, Roberto (1958), “Monografía del Estado de Baja California”, en Memoria del Primer Congreso de Historia Regional de Baja California, Dirección de Acción Cívica y Cultural del Gobierno de Baja California, Mexicali, B.C.

Martínez Sandoval, Rodrigo (1987), "Los distrito de riego y su impacto en el desarrollo de la frontera norte (apuntes preeliminares)", ponencia presentada en el primer Encuentro sobre Problemas del Campo en el Noroeste de México y el Suroeste de los Estados Unidos, efectuada en la ciudad deCuliacán, Sinaloa, los días 13 al 16 de enero .

Moreno Mena, JoséA. (1994a), “La conformación histórica del mercado de trabajo agrícola en el valle de Mexicali", revista Estudios Fronterizos, M exicali, Instituto del nvestigaciones Sociales, núm.. 33, enero-junio, UABC. (1994b), "Una aproximación a las características actuales de la mano de obra agrícola en el valle de Mexicali", revista Estudios Fronterizos, M exicali, Instituto de Investigaciones Sociales, núm. 34, julio-diciembre, UABC. (1994c), "Contaminación por plaguicidas en la frontera norte", revista Semillero de I deas, UABC, número 5, año 2, enero-marzo de 1994.

(1993), "Los plaguicidas en los distritos deriego del noeroeste", Diálogos del D iario 29, Tijuana, B.C., 2 de julio. 
Rocha Valencia, Félix (1992), "Notas sobre la mosquita blanca", diario La Voz de la Frontera, suplemento "Voz a Nova", 19 de abril.

Samplin Program for the determination of multimedia transport and fate of pesticide concentractions in thel mperial and M exicali valleys, mimeo, 1989.

Sanchez Ramírez, Óscar (1990), "Crónica agrícola del valle de Mexicali", UABC.

SARH (1992), revista Sanidad V egetal, Mexicali, B.C.

Secretaría de Fomento Agríco-la del Estado de Baja California (2000)

www.bajacalifornia.gob.mx. (2004), Estadísticas agrícolas.

Secretaría de Salud (2000), Delegación Baja California. Dirección

General de Salud Ambiental.

Stamatis, Martha (1992), A gricultura e inversión extranjera en el valle de

M exicali: la producción de hortalizas de exportación, tesis de maes-

tría, México, Universidad de Chapingo.

Stamatis, Martha (1987), “El valledeM exicali: agricultura einversion extranjera” (1901-1986)", Estudios Fronterizos, Mexicali, IIS-UABC, año $V$, vol. V, núms. 12-13.

Valdés Márquez, M., M. M. Laresy F. Bustamante(2002), “Plaguicidas organoclorados y BPC's en mujeres que han procreado hijos con gastrosquisis", mimeo, Ensenada, B.C.

Sagarpa (2002), D el egación B.C. Distrito de D esarrollo Rural 002, Río CoIorado, Dirección General de Salud A mbiental, Cicoplafest.

Entrevista a Trinidad García (1994) en el Ejido Cucapah M estizo, valle de Mexicali, Mexicali, B.C.

Entrevista al doctor Carlos Enrique Peña Limón (1993), director del Departamento de Investigación Científica y Tecnólogica de la Universidad deSonora, Hermosillo, Sonora, 26 de diciembre.

Artículo recibido el 12 de mayo de 2005 Artículo aprobado el 10 de octubre de 2005 
